\title{
A case of paradoxical cerebral infarction by spraying thrombus in the catheter for dialysis
}

\author{
Seiji Hashimoto ${ }^{1 *}$ (D) Hiroyoshi Sasaki ${ }^{2}$, Hiroshi Matsuura², Takao Koike ${ }^{2,3}$ and Tomoaki Haga ${ }^{4}$
}

\begin{abstract}
Background: The authors herein report a case of paradoxical cerebral infarction by spraying thrombus in the catheter for dialysis. The clinical manifestations of paradoxical cerebral infarction are nonspecific, and the diagnosis is sometimes difficult to establish. Patients with paradoxical cerebral infarction may present with neurologic abnormalities or features suggesting arterial embolism. And paradoxical cerebral infarction has been associated with foramen ovale (PFO).

Case presentation: A multi-lumen catheter for dialysis was inserted into the right internal jugular vein. After injecting heparin-added physiological saline solution to confirm the patency of the catheter, paradoxical cerebral infarction developed. Detailed examination revealed PFO. PFO patent is a common condition that affects one in four people.

Conclusion: The size of multi-lumen catheter for dialysis is relatively large. We learned from this case that the use of multi-lumen catheter poses a potential risk of paradoxical infarction. Clinicians should be aware that central venous catheter is a risk factor for paradoxical cerebral infarction.
\end{abstract}

Keywords: Paradoxical cerebral infarction, Catheter, Foramen ovale

\section{Background}

Paradoxical cerebral infraction is a pathological condition caused by paradoxical embolism, in which clots or air travel from the venous side to the arterial side via the right-to-left shunt due to patent foramen ovale, anomaly of pulmonary artery, atrial septal defect, or ventricular septal defect, etc. [1]. In general, embolism does not occur even if there is a hole in the septal wall because blood pressure in the arterial side is higher than that in the venous side. A rightto-left shunt requires transient or permanent increase of the pressure in the right ventricle and atrium compared to the left side. The incidence of conditions such as anomaly of pulmonary artery and atrial septal defect is low, and their presence is usually recognized when patients receive medical treatment. Therefore,

\footnotetext{
*Correspondence: seijinih@med.hokudai.ac.jp

'NTT East Japan Sapporo Hospital Department of Nephrology, South 1, West

15, Chuou-ku-ku, 060-0061, Sapporo, Japan

Full list of author information is available at the end of the article
}

sudden onset of paradoxical infarction is considered to be extremely rare.

On the other hand, patent foramen ovale affects approximately $25 \%$ of healthy adults [2]. It is not a rare condition. However, abnormalities including paradoxical infarction do not develop unless a right-to-left shunt is present, as described above. Therefore, most patients with patent foramen ovale are asymptomatic. We experienced a case of paradoxical cerebral infarction caused by a double-lumen catheter for dialysis. The size of multi-lumen catheter for dialysis is relatively large.

\section{Case presentation}

The patient was a 40-year-old woman who had been treated for ulcerative colitis at our department of gastroenterological medicine. She was admitted to our hospital because of poor control of disease and was scheduled for granulocyte apheresis (GCAP). Since it was difficult to secure vascular access because the patient's blood vessels were thin, a double-lumen catheter (GamCath ${ }^{\circ}$ 
HighFlow Double-Lumen Catheter Kit GDHK-1315) for dialysis was inserted into the right internal jugular vein for blood access. GCAP was performed twice a week, and there were no problems until after the completion of the second treatment.

Two days after the second GCAP, maintenance of catheter was performed in the ward. The maintenance consisted of confirmation of patency and injection of heparin into the catheter, which was a routine procedure for patients with long-term indwelling catheter for the prevention of catheter occlusion. A nurse extracted the contents of the catheter using an empty 5 - $\mathrm{mL}$ syringe. Approximately $4 \mathrm{~mL}$ of fluid and a tiny amount of thrombi were aspirated. Then, $5 \mathrm{~mL}$ of a heparinized solution was injected into the catheter using a Heparin $\mathrm{Na}$ Lock 10 -units $/ \mathrm{mL}$ syringe (Otsuka Pharmaceutical). After confirming the patency, the catheter was enclosed. Immediately after this, the patient complained of respiratory discomfort, numbness in the left side of the body, and visual field abnormality. The patient was referred to a local neurosurgery clinic. Computed tomography $(\mathrm{CT})$ and magnetic resonance imaging (MRI) of the brain showed no obvious abnormalities and her subjective symptoms improved, and the patient was followed up without surgery. The patient had no history of diseases, except for ulcerative colitis. In particular, she had no history of arteriosclerotic disease.

Seven days later, the catheter maintenance procedure was carried out again while the patient was in a sitting position because she was talking to her friend. Immediately after this, the patient complained of tightness in the chest, numbness in the left side of the body, and rigidity in the right upper extremity, and a sense of feeling bad. She was monitored on an electrocardiogram and suspected as having ST elevation. An emergent cardiac catheterization was performed and showed no evidence of coronary artery disease. Pulmonary computed tomography was performed, and the scan showed no abnormalities. After cardiac catheterization, the patient was referred to the Department of Neurosurgery for detailed neurological examination.

The brain magnetic resonance imaging scan showed a punctuated and patchy high signal intensity, suggesting cerebral infarction in the territories of both anterior cerebral arteries, both posterior cerebral arteries, and the right posterior central gyrus cortex (Fig. 1). The patient was suspected to have an underlying problem because she had previously had a similar episode. Transcranial Doppler ultrasound was performed. When Valsalva strain was released, microbubbles appeared. Echocardiography was performed twice and showed no abnormalities. Therefore, abnormalities such as patent foramen ovale were suspected. Contrast transesophageal echocardiography was performed. Immediately after a Valsalva strain, echocardiography showed appearance of microbubbles in a same manner (Fig. 2). Subsequently, the presence of patent foramen ovale was confirmed (Fig. 3). Based on these findings, the patient was diagnosed with paradoxical cerebral infarction due to patent foramen ovale. However, the tissue plasminogen activator was not administered because more than $4 \mathrm{~h}$ had passed since the onset of cerebral infarction. After anticoagulant therapy, the patient underwent rehabilitation. Her subjective symptoms improved after treatment of cerebral infarction.

\section{Discussion}

Paradoxical cerebral infarction is a condition caused by paradoxical embolism, in which obturators travel from the venous system to the arterial system via the right-to-left shunt. The requirements for this condition are twofold: "communication between the venous and arterial systems" and "increased pressure in the venous system." Paradoxical cerebral infarction does not occur if one of the requirements is lacking.

Patent foramen ovale is a common condition that affects one in four people [3], and it is difficult to detect with conventional echocardiography. In most cases, transesophageal echocardiography is required for the diagnosis $[4,5]$. It is well known that patent foramen ovale is a risk factor for cerebral infarction; the resulting conditions are referred to as cryptogenic strokes or strokes of unclear origin. Treatments for patent foramen ovale, such as closure surgery, are under discussion [6]. More importantly, many cases of foramen ovale are asymptomatic, as was the present case, and the condition is therefore underdiagnosed.

Paradoxical cerebral infarction is a well-known disease among experienced neurosurgeons. As expected, many reported cases of paradoxical cerebral infarction were caused by a patent foramen oval because of its high incidence. There are also several reported cases of cerebral infarction as a catheter-related complication. An air embolism is frequently found to be the cause. In particular, there are many reported cases of air migration at the time of catheter removal. Previous reports on paradoxical cerebral infarction due to catheter placement are shown in Table 1. There have been various cases of air embolism, and they are typically associated with multi-syringe catheters placed into the internal jugular veins.

In the present patient, the presence of a patent foramen oval was confirmed. We considered that the cerebral infarction was caused by catheter placement 

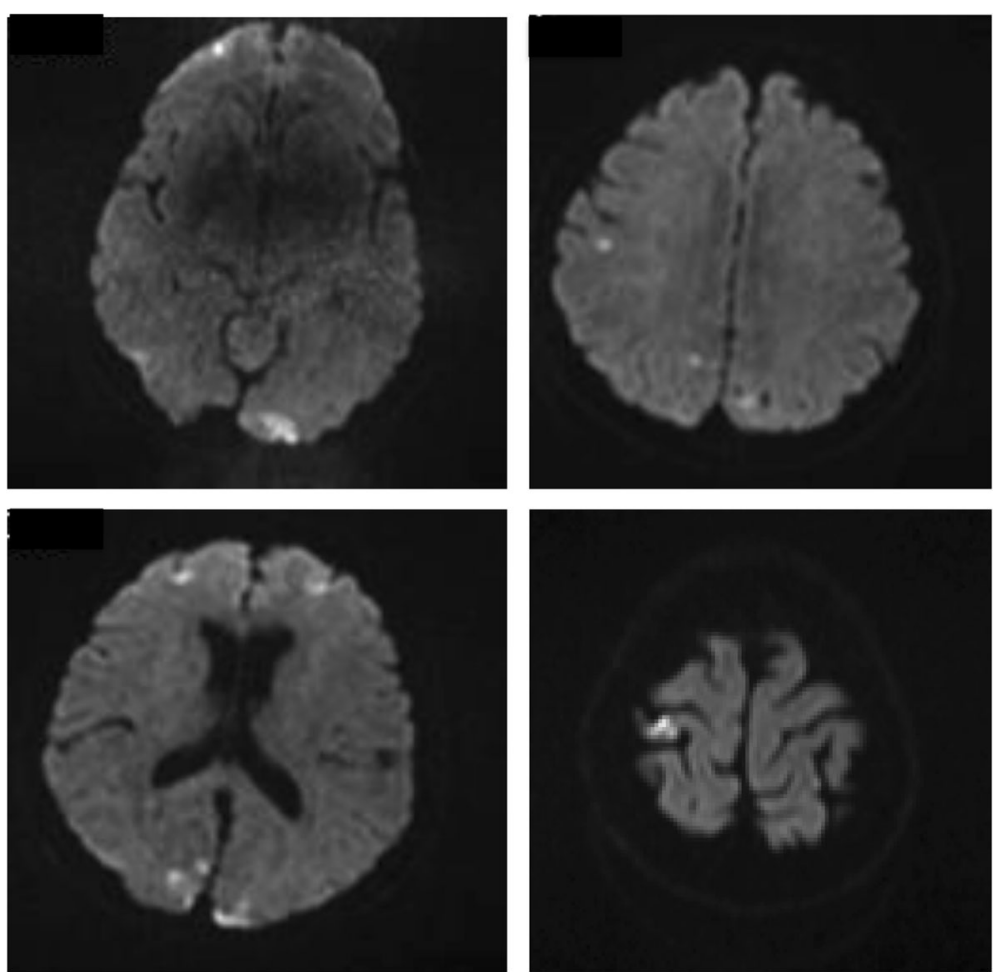

Fig. 1 Brain magnetic resonance imaging: diffusion-weighted magnetic resonance imaging
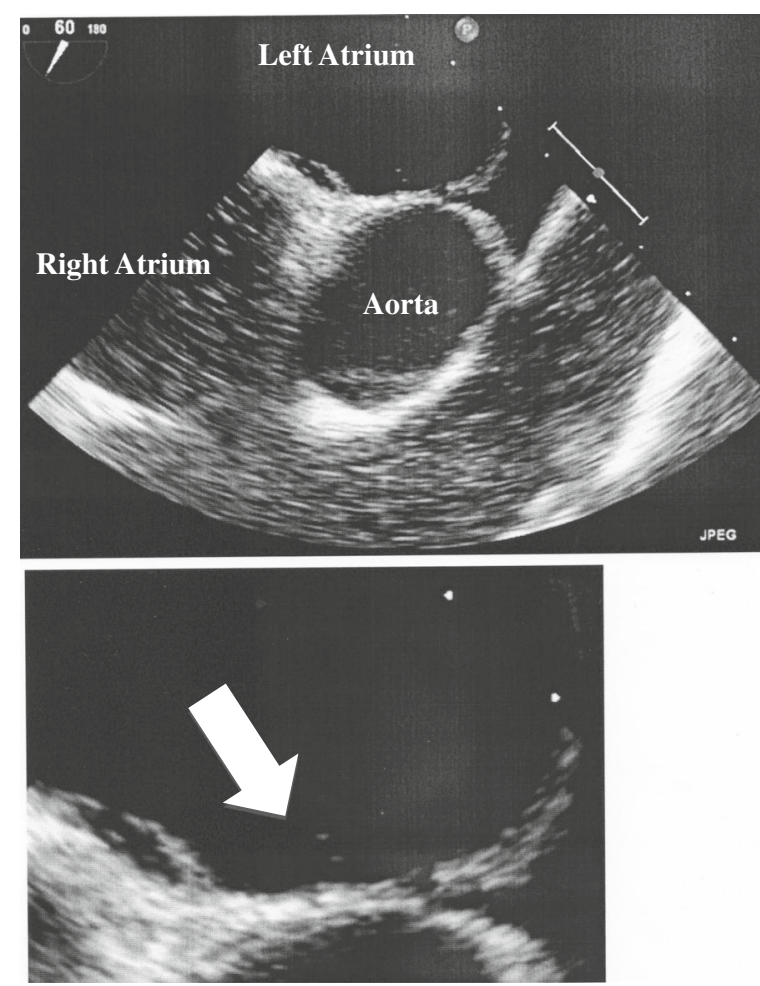

Fig. 2 Contrast transesophageal echocardiography after a valsalva strain. Numerous microbubbles are present in the right atrium. Bottom A magnified view of the upper image. The arrow indicates two microbubbles that traveled to the left atrium because the patient showed similar symptoms after the catheter maintenance procedures. The patient discussed herein had no history of diseases, such as atrial fibrillation, that can cause arterial thrombosis. She also had no history of deep vein thrombosis or predisposing factors to venous thrombosis. To our knowledge, this is the first case of a patient with paradoxical cerebral infarction that was clearly caused by a flash of thrombi in the catheter.

In general, small thrombus formation does not lead to cerebral infarction of the systemic arterial system. Catheters are usually placed in the veins. Even if a patent foramen ovale is present, blood clots do not travel to the arterial system unless a right-to-left shunt is present, which occur only when venous pressure exceeds arterial pressure. It is therefore important to keep the blood pressure in the right atrium and ventricle well controlled during catheter procedures to prevent cerebral infarction. In general, blood pressure in the right atrium and ventricle is elevated by straining, lifting heavy baggage, defecating, sexual intercourse, changing position from lying to sitting, coughing, and sneezing. Therefore, it is important to avoid such conditions during catheter procedures. In the present case, the second catheter maintenance procedure was carried out while the patient was 

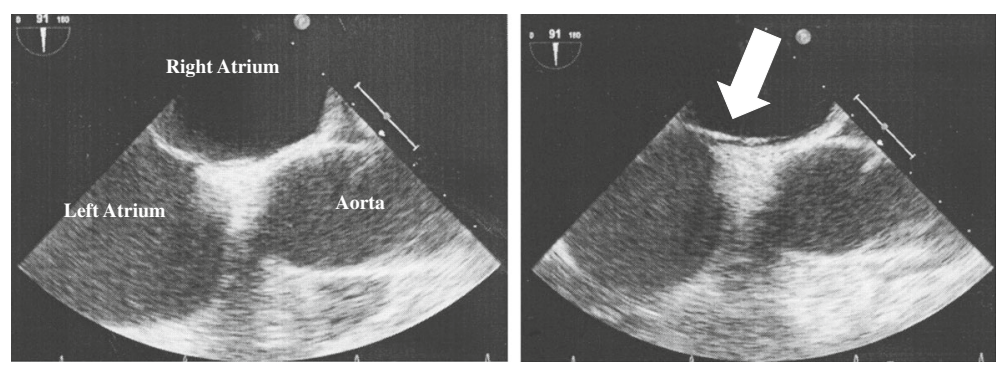

Fig. 3 Contrast transesophageal echocardiography. Left PFO closed. Right PFO is open (arrow)

sitting, which might have been a cause of cerebral infarction. As shown in Table 1, a higher percentage of patients underwent the procedure while sitting or standing, which is a risk of thrombosis, as confirmed by a study that examined the effects of body position during operation. Therefore, we conclude that catheter management should not be performed with patients in a sitting or standing position.

We consider it important to remove small thrombi in the catheter during catheter use and maintenance. Thrombi should be aspirated as completely as possible, and flushing them should be avoided as much as possible. There is no established consensus on regular catheter maintenance procedures, such as heparin injection, for the prevention of catheter occlusion. The National Kidney Foundation Kidney Disease Outcomes Quality Initiative (NKF KDOQI) guidelines, the European Renal Best Practice guidelines, and the Canadian Society of
Nephrology guidelines do not stipulate whether such management is needed. On the other hand, the Japanese Society for Dialysis Therapy Guidelines for Vascular Access Construction and Repair for Chronic hemodialysis (2005) state that "Anticoagulant therapy such as heparin lock should be performed only on the day of hemodialysis because infection rate is lower in this manner compared with daily administration. However, this is not a strict rule because some patients require anticoagulant therapy such as heparin lock also on the day without hemodialysis for the prevention of thrombus formation in the catheter." [7]. From the viewpoint of prevention of catheter infection, the Centers for Disease Control and Prevention (CDC) guidelines stipulate as follows [8]: "Do not routinely use anticoagulant therapy to reduce the risk of catheter-related infection in general patient populations" (Category II). They recommended that anticoagulant therapy should not be performed for the prevention of

Table 1 Previous reports on paradoxical cerebral infarction due to catheter placement

\begin{tabular}{|c|c|c|c|c|c|c|}
\hline $\mathrm{CN}$ & Author & Emboli & Operation & Catheter type & Catheter position & Position at treatment \\
\hline [9] & Kariya S & Air & Removal & Single & Right internal jugular vein & Supine position \\
\hline [10] & Eichhorn V & Air & Removal & Triple lumen (7 Fr) & Right internal jugular vein & Sitting in an upright position \\
\hline [11] & Copetti R & Air & Insert & NC & Right internal jugular vein & Supine position \\
\hline [12] & Khan $\mathrm{H}$ & Air & Removal & Triple lumen & Right internal jugular vein & Sitting in an upright position \\
\hline [13] & Petrea RE & Coagulus & NC & PICC & $\begin{array}{l}\text { Superior vena cava prolapsing into } \\
\text { the right atrium }\end{array}$ & NC \\
\hline [14] & Bowles PF & Air & Removal & Pulmonary artery (PA) catheter & Right internal jugular vein & Supine position \\
\hline [15] & Wu YC & Air & Removal & NC & NC & NC \\
\hline [16] & Levy SD & Air & Removal & 14 Fr catheter & NC & Trendelenburg position \\
\hline [17] & Yu AS & Air & After HD & Double lumen & The right subclavian vein & NC \\
\hline [18] & Zuha R & Air & Removal & NC & NC & $\mathrm{NC}$ \\
\hline [19] & Batsis JA & Coagulus & NC & Single & Right internal jugular vein & Supine position \\
\hline [20] & De Jong A & Air & Removal & NC & $\mathrm{NC}$ & $\mathrm{NC}$ \\
\hline [21] & Cardim N & Coagulus & NC & Permanent CVC & Superior vena cava & NC \\
\hline [22] & Han SS & Air & Insert & $\mathrm{NC}$ & Right internal jugular vein & NC \\
\hline [23] & Suzuki S & Coagulus and air & Removal & Double lumen & Right internal jugular vein & Supine position \\
\hline [24] & Biller J & Coagulus & NC & Swan-Ganz catheter & NC & NC \\
\hline
\end{tabular}

CN citation number, NC not clear, PICC peripherally inserted central catheter, HD hemodialysis, CVC central venous catheter 
infection. Given that the effectiveness of anticoagulant therapy for the prevention of catheter occlusion has not been established, it may be appropriate not to perform anticoagulant therapy, except for special cases, in order to reduce the risk of infection and paradoxical cerebral infarction. In addition, it is important to consider the possibility of paradoxical cerebral infarction when a patient shows symptoms of cerebral infarction or coronary artery infarction after catheter maintenance procedure, etc.

\section{Conclusion}

We learned from this case that the use of multi-lumen catheter poses a potential risk of paradoxical infarction. Specialists in dialysis and cardiovascular disease and neurosurgeons should be aware of the risk of paradoxical cerebral infarction. Clinicians should be aware the risk of paradoxical cerebral infarction in patients with indwelling central venous catheter.

\section{Abbreviations}

CN: Citation number; CT: Computed tomography; CVC: Central venous catheter; GCAP: Granulocyte apheresis; HD: Hemodialysis; MRI: Magnetic resonance imaging; NC: Not clear; PFO: Foramen ovale; PICC: Peripherally inserted central catheter

\section{Acknowledgements}

The authors would like to express our sincere thanks to the medical staffs of Nakamura Memorial Hospital, Sapporo, Japan.

\section{Funding}

Not applicable. Our manuscript does not contain funding.

\section{Availability of data and materials}

The data and materials were all included in the manuscript.

\section{Ethics approval and consent to participate}

Written informed consent was obtained from the patient after a detailed explanation of the objectives of the study. The patient requested strongly that the report be published to aid in the prevention of paradoxical cerebral infarction in patients with indwelling catheter.

\section{Authors' contributions}

$\mathrm{SH}$ is responsible for the manuscript. HS, HM, and TK are staff of the Medical Safety Management Office and conducted a cause investigation. TH was in charge of actual diagnosis and treatment. All authors read and approved the final manuscript.

\section{Authors' information}

$\mathrm{SH}$ is a Director of NTT East Japan Sapporo Hospital Department of Nephrology, Sapporo, Japan. HS works at the Department of Medical Safety at NTT East Japan Sapporo Hospital, Sapporo, Japan. HM is a Deputy Director of NTT East Japan Sapporo Hospital. TK is an Emeritus Professor of Hokkaido University and Director of NTT East Japan Sapporo Hospital. TH is the Director of Makamura Memorial Hospital Department of Cardiology, Sapporo, Japan.

\section{Consent for publication}

Written informed consent was obtained from the patient for publication of this case report and the accompanying images.

\section{Competing interests}

The authors declare that they have no competing interests.

\section{Publisher's Note}

Springer Nature remains neutral with regard to jurisdictional claims in published maps and institutional affiliations.

\section{Author details}

${ }^{1} \mathrm{NTT}$ East Japan Sapporo Hospital Department of Nephrology, South 1, West 15, Chuou-ku-ku, 060-0061, Sapporo, Japan. ${ }^{2} N T T$ East Japan Sapporo Hospital Department of Medical Safety, Sapporo, Japan. ${ }^{3}$ Department of Medicine II, Hokkaido University Graduate School of Medicine, Sapporo, Japan. ${ }^{4}$ Nakamura Memorial Hospital Department of Cardiology, Sapporo, Japan.

Received: 1 May 2017 Accepted: 7 August 2017

Published online: 23 October 2017

\section{References}

1. Windecker S, Stortecky S, Meier B. Paradoxical embolism. J Am Coll Cardiol. 2014;64:403-15.

2. Homma S, Di Tullio MR. Patent foramen ovale and stroke. J Cardiol. 2010;56: 134-41.

3. Sommer RJ. Patent foramen ovale: where are we in. 2009. Am J Ther. 2009; 16:562-72.

4. Dalen JE, Alpert JS. Cryptogenic strokes and patent foramen ovales: what's the right treatment? Am J Med. 2016;129:1159-62.

5. Mojadidi MK, Bogush N, Caceres JD, et al. Diagnostic accuracy of transesophageal echocardiogram for the detection of patent foramen ovale: a meta-analysis. Echocardiography. 2014;31:752-8.

6. Furlan AJ, Reisman M, Massaro J, et al. Closure or medical therapy for cryptogenic stroke with patent foramen ovale. N Engl J Med. 2012:366:991-9.

7. Ohira S, Naito H, Amano I. 2005 Japanese Society for Dialysis Therapy Guidelines for vascular access construction and repair for chronic Hemodialysis. Ther Apher Dial. 2006;10:449-62.

8. O'Grady NP, Alexander M, Burns LA, et al. Guidelines for the prevention of intravascular catheter-related infections. Clin Infect Dis. 2011;52:e162-93.

9. Kariya S, Tanigawa N, Komemushi A, et al. Asymptomatic paradoxical and symptomatic pulmonary air embolism during central venous catheter insertion. Jpn J Radiol. 2010;28:473-5.

10. Eichhorn $\mathrm{V}$, Bender $\mathrm{A}$, Reuter DA, et al. Paradoxical air embolism from a central venous catheter. Br J Anaesth. 2009;102:717-8.

11. Copetti R De Monte A. Rapid echocardiographic diagnosis of suspected paradoxical gas embolism after central venous catheter placement in the upright position. Echocardiography. 2005;22:695-6.

12. Khan $\mathrm{H}$, Zaidi A. Paradoxical air embolism following central venous catheter removal. BMJ Case Rep. 2013;26:2013.

13. Petrea RE, Koyfman F, Pikula A, et al. Acute stroke, catheter related venous thrombosis, and paradoxical cerebral embolism: report of two cases. J Neuroimaging. 2013;23:111-4.

14. Bowles PF, Lear C, Maccario M, et al. Paradoxical air embolism and neurological insult during removal of a pulmonary artery catheter introducer. BMJ Case Rep. 2014;10:2014.

15. Wu YC, Liu HP, Liu YH, et al. Paradoxical cerebral air embolism after removal of a central venous catheter: case report. Chang Gung Med J. 2000;23:706-10.

16. Levy SD, Oren-Grinberg A, McSparron JI. Paradoxical air embolus after removal of a central venous catheter. Ann Am Thorac Soc. 2016;13:1856-7.

17. Yu AS, Levy E. Paradoxical cerebral air embolism from a hemodialysis catheter. Am J Kidney Dis. 1997;29:453-5.

18. Zuha R, Price T, Powles $R$, et al. Paradoxical emboli after central venous catheter removal. Ann Oncol. 2000:11:885-6.

19. Batsis JA, Craici IM, Froehling DA. Central venous catheter thrombosis complicated by paradoxical embolism in a patient with diabetic ketoacidosis and respiratory failure. Neurocrit Care. 2005;2:185-8.

20. De Jong $A$, Jung $B$, Cissé $M$, et al. Gas embolism: another story related to a central venous catheter withdraw. Ann Fr Anesth Reanim. 2011;30:366-8.

21. Cardim N, Toste J, Carvalho V, et al. Playing games with a thrombus: a dangerous match. Paradoxical embolism from a huge central venous catheter thrombus: a case report. Cardiovasc Ultrasound. 2010;16(8):6.

22. Han SS, Kim SS, Hong HP, et al. Massive paradoxical air embolism in brain occurring after central venous catheterization: a case report. J Korean Med Sci. 2010;25:1536-8

23. Suzuki S, Sanui M. A case of paradoxical cerebral thromboembolism after removal of a central venous catheter. Can J Anaesth. 2007:54:326-7.

24. Biller J, Adams HP Jr, Johnson MR, et al. Paradoxical cerebral embolism: eight cases. Neurology. 1986;36:1356-60. 